\title{
Bienestar universitario virtual, una estrategia para el fortalecimiento de la comunidad unadista con el uso de las TICs.
}

\author{
Mauricio Perdomo Vargas ${ }^{1}$ \\ Víctor Fernando Cañón Rodríguez ${ }^{2}$
}

\begin{abstract}
Resumen
La enseñanza online demanda el desarrollo de diferentes actividades de bienestar en un campus virtual con la utilización de herramientas propias de las TICs y la Web 2.0, esto permite generar un ambiente de aprendizaje virtual mucho más integral, dinámico e interesante. Según esto y como conclusiones finales del presente estudio, se entrega a consideración de la comunidad académica de la UNAD (Universidad Nacional Abierta y a Distancia) una propuesta estructural donde se identifican los elementos, tipos de interacción y los subsistemas del Sistema de Bienestar Universitario Virtual-BUV- que se propone como resultado final de la investigación.
\end{abstract}

Palabras clave: bienestar, comunicación, mediación virtual, moodle, tecnología.

\footnotetext{
1 Ingeniero de sistemas con énfasis en telecomunicaciones, Universidad Cooperativa de Colombia. Máster Of Arts In Education - Online Education UNAD Florida. Diplomado en: Tutor virtual UNAD, Director virtual de la UNAD, Diseñador de material didáctico UNAD y Diseñador de OVAS UNAD. Tutor TC ECBTI Universidad Nacional Abierta y a Distancia UNAD CERES, La Plata, Huila. Correo: mauricio.perdomo@unad.edu.co - Colombia.

2 Ingeniero de sistemas Universidad Cooperativa de Colombia. Especialista en Pedagogía paara el Aprendizaje Autónomo de la UNAD. Máster Of Arts In Education - Online Education UNAD Florida. Diplomado en Redes y telecomunicaciones, Tutor virtual de la OEA y la Universidad del Rosario, Director virtual de la UNAD y Diseñador de OVAS UNAD. Tutor TC ECBTI Universidad Nacional Abierta y a Distancia UNAD CEAD Tunja Boyacá. Correo: victor.canon@unad.edu.co - Colombia.
} 


\title{
Online university welfare - a strategy to strengthen UNAD's community through the use of TIC's
}

\begin{abstract}
Online education requires the development of different welfare activities in an online campus with the use of TIC and Web 2.0's tools. This will generate a far more comprehensive, dynamic and interesting virtual learning environment. According to this and as a final result of the present research, a structural proposal is put under UNAD's community's consideration, which identifies the elements, types of interaction and subsystems of the Virtual University Welfare System —BUV.
\end{abstract}

Key Words: welfare, communication, virtual mediation, moodle, technology.

Recibido: 22 de junio 2011

Aceptado: 18 de agosto 2011

\section{Introducción}

El proyecto Bienestar Universitario Virtual, para el fortalecimiento de la comunidad Unadista con el uso de las TICs, surge de una necesidad y un problema sentido y latente en la comunidad académica de la UNAD, donde paradójicamente se maneja un sistema de educación a distancia de tipo AvDtDe (Alta virtualidad, Diferentes tiempos, Diferentes espacios), pero no se potencia el modelo como de formación integral sino que solo se maneja como un modelo académico puro apoyado por las TICs.

La necesidad de contar con un escenario de formación integral donde se rescaten valores, culturas y tradiciones, además de potenciar a un individuo en diferentes campos, como ser integral, ampliamente social e interactivo en su contexto, construye la justificación de la investigación propuesta.

\section{Metodología}

Ubicación geográfica: esta investigación fue realizada dentro del contexto de la comunidad universitaria de la Universidad Nacional Abierta y a Distancia 
UNAD-, la cual está distribuida en 8 zonas propias de la geografía Colombiana y con una sede en los Estados Unidos de América; su sede principal está en la ciudad de Bogotá en la calle 14 sur Número 14-23 Barrio Restrepo.

Tipo de estudio: el presente proyecto se enmarcó dentro de un contexto metodológico propio de un estudio de tipo mixto, ya que como lo define Zorrilla: «la investigación mixta es aquella que participa de la naturaleza de la investigación documental y de la investigación de campo» (1993:43). Según esto se definieron dos fases para el desarrollo del análisis de datos en el proyecto.

La primer fase contempló el análisis de diferentes tipos de información documental relacionadas con el problema, la cual se obtuvo de fuentes como: libros, revistas, periódicos, memorias, anuarios, registros, códices, constituciones, artículos, investigaciones o estrategias de otras instituciones y otros documentos que abordan de forma directa y específica el tema objeto de estudio.

Para la segunda fase se realizó un proceso de «trabajo de campo», donde se realizó una observación y exploración directa sobre el problema o contexto investigado. Para esto se llevó a cabo el diseño y aplicación de un instrumento de recolección de datos, el cual fue procesado para desarrollar una labor rigurosa de análisis e interpretación de los resultados que se generaron al aplicar el instrumento.

Variables: dentro del proyecto se concibieron dos variables las cuales ayudaron a delimitar el problema y el norte de la investigación, la variable No. 1 se basaba en conocer las características de tipo estructural, psicosocial, de gestión, de innovación y acreditación de alta calidad que debe poseer un sistema de bienestar universitario virtual. La variable $\mathrm{N}^{0} 2$ requería identificar y determinar las necesidades de la comunidad universitaria de la UNAD, con base en los programas o servicios de bienestar universitario virtual que esta misma comunidad requiere para su desarrollo y formación integral.

Población y muestra: la población del proyecto se enmarcó dentro de la comunidad universitaria de la UNAD. A su vez se definió una muestra representativa consistente en 1.530 estudiantes y 20 tutores de la universidad pertenecientes a la Red Académica de los cursos académicos Teoría General de Sistemas, Diseños de Sitios Web y Redes locales Básico, además de 10 funcionarios administrativos de diferentes CEAD de la UNAD. Es importante también destacar que en consecuencia con el tipo de estudio mixto con el que se abordó la investigación, se analizaron 16 documentos digitales relacionados con el problema de la investigación.

Instrumento: se diseñó una encuesta como primer instrumento piloto, con la cual se realizó en primera instancia una validación analítica de la misma. Dicha validación se realizó entre el 15 y el 20 de Octubre de 2010, donde se aplicó el primer cuestionario a 30 personas de la comunidad universitaria para realizar este proceso. Esta encuesta se diseñó con preguntas cerradas debido a que son más 
objetivas, válidas y confiables, además la misma contempló el uso de respuestas sistémicas que permitieron «configurar un conjunto de categorías de respuesta que se mantiene constante de manera que las respuestas de los sujetos a los estímulos se registra sobre un conjunto predeterminado de pre-respuestas o respuestas pre-codificadas» (Gallardo y Moreno, 1999:78). Procedimientos: se tuvieron en cuenta los siguientes aspectos relacionados con la administración del instrumento:

Diseño de preguntas: se planteó la construcción de 17 preguntas para la encuesta de acuerdo con las características del instrumento en cuanto a tipo de preguntas y tipo de respuestas las cuales se definieron anteriormente en el documento.

Muestra poblacional: esta muestra se determinó de acuerdo a la población total de los tres cursos y los administrativos, la cual fue de 1.550 personas como población total más las variables contempladas de acuerdo a la fórmula para hallar la muestra:

$$
\begin{aligned}
& n=\quad N * Z_{\alpha}^{2} p^{*} q \\
& e^{2}(N-1)+Z_{\alpha}^{2} * p * q
\end{aligned}
$$

De lo anterior tenemos que:

$\mathrm{N}=$ Total de la población

$\mathrm{Z} \square 2=1.962$ (considerando una seguridad del 95\%)

$\mathrm{p}=$ proporción esperada (en este caso $8 \%=0.08)$

$\mathrm{q}=1-\mathrm{p}($ en nuestro caso $1-0.08=0.92)$

$\mathrm{e}=$ Margen de error (en este caso deseamos un 5\%)

Aplicando la fórmula se tiene lo siguiente para la aplicación de la encuesta:

$$
\begin{aligned}
& n=\frac{1550 * 1.96^{2} * 0.05 * 0.092}{0.05^{2}(1550-1)+1.96^{2} * 0.08 * 0.92} \\
& n=115
\end{aligned}
$$

Diseño de encuesta virtual en Google: para el diseño del cuestionario se utilizó el módulo de cuestionarios de Google el cual fue enlazado a los tres cursos virtuales para su respectivo diligenciamiento.

Análisis de los resultados arrojados por el instrumento: se procedió después de la aplicación del instrumento de recolección de datos, a realizar una labor de análisis e interpretación de los mismos, fundamentada en una descripción de tipo cuantitativa y cualitativa de los datos arrojados por el instrumento. 
Método de análisis de datos: para la interpretación de los datos arrojados por el instrumento aplicado, se utilizó el método cuantitativo por medio de un análisis estadístico en el cual se implementaron las técnicas de: distribución de frecuencias; porcentajes (\%); promedios, desviación estándar; gráficos de barras, de sectores, histogramas, a su vez estos resultados se usaron como insumo para el desarrollo de un breve análisis cualitativo.

Resultado de análisis de datos: la lectura de los datos se realizó en dos partes, una fue el diagnóstico inicial donde nos arrojó datos generales e informativos y una segunda parte: la estructura del bienestar universitario donde arrojó datos de una posible estructura amigable para los usuarios dentro de un portal web. A continuación y con miras a la optimización del contenido del presente artículo solo se presentan los datos más representativos arrojados como resultados finales por el instrumento aplicado:

\section{Resultados}

1. Pregunta de caracterización, su rol dentro de la comunidad universitaria en la UNAD es:

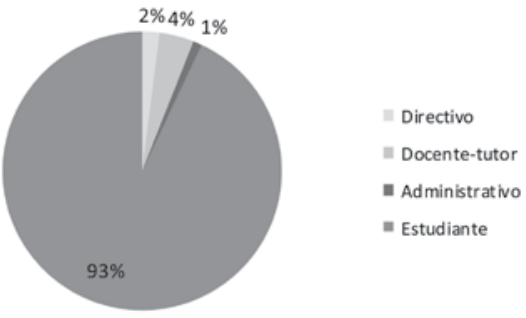

\begin{tabular}{|l|l|l|}
\hline Directivo & 5 & $2 \%$ \\
\hline Docente-tutor & 9 & $4 \%$ \\
\hline Administrativo & 2 & $1 \%$ \\
\hline Estudiante & 230 & $93 \%$ \\
\hline
\end{tabular}

Figura 1. Presentación gráfica, resultados pregunta No. 1

2. Actualmente la UNAD cuenta con dos Mediaciones Pedagógicas (Tradicional y Virtual), Según esto ¿cuál de las mediaciones utiliza usted al interior de la universidad?

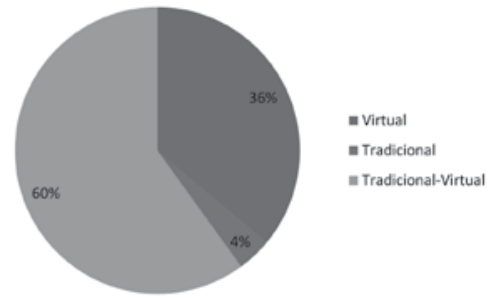

\begin{tabular}{|l|l|l|}
\hline Virtual & 88 & $36 \%$ \\
\hline Tradicional & 10 & $4 \%$ \\
\hline Tradicional-Virtual & 148 & $60 \%$ \\
\hline
\end{tabular}

Figura 2. Presentación gráfica, resultados pregunta No. 2 
En la respuesta a la pregunta número 2 podemos observar que hay solo un $10 \%$ interactuando académicamente de forma tradicional lo que indica que si tomamos el ítem 1 y 3 podríamos decir que el 90\% de nuestra comunidad universitaria está realizando al menos una actividad de forma virtual, en el caso de los estudiantes al menos un curso virtual, es por ello que el gran porcentaje de encuestados corresponde a estudiantes.

3. ¿En el centro al cual usted está matriculado, actualmente desarrollan programas de bienestar universitario?

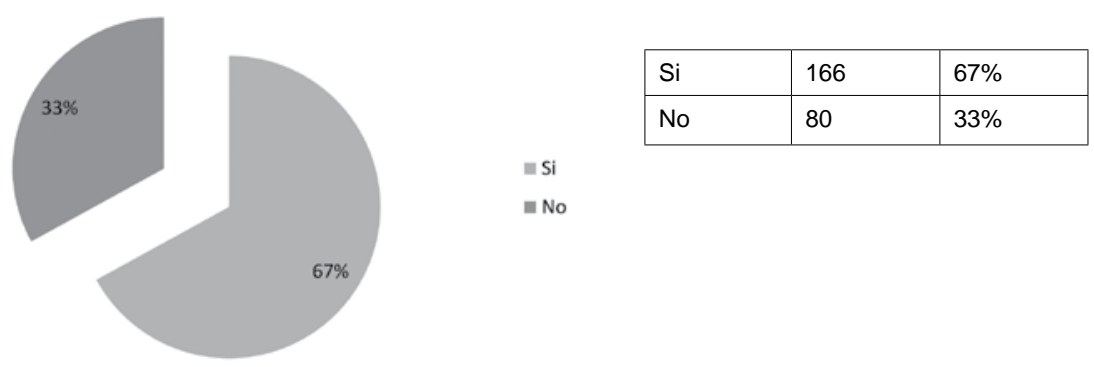

Figura 3. Presentación gráfica, resultados pregunta No. 3

Sin lugar a dudas podemos observar en la respuesta a la pregunta 3 , que hay un gran porcentaje (33\%) de centros que en este momento carecen de un bienestar universitario, algo preocupante ya que se está restringiendo los derechos de los estudiantes en este sentido, algo que podría ser resuelto de alguna forma con la propuesta de BUV. Se debe tener en cuenta que esta población, que aún no tiene aplicaciones de servicios propios del BUV, puede corresponder a centros pequeños como Unidades de Desarrollo Regional (UDR) o a Centros Regionales de Educación Superior (CERES).

4. Según lo expuesto anteriormente, ¿considera usted que la UNAD debe ofrecer un sistema de bienestar universitario virtual, que permita garantizar el acceso y la cobertura de los programas de bienestar universitario a toda la comunidad Unadista?

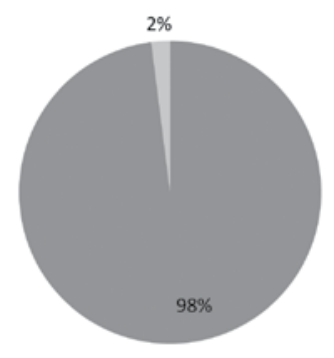

\begin{tabular}{|l|l|l|}
\hline $\mathrm{Si}$ & 240 & $98 \%$ \\
\hline No & 6 & $2 \%$ \\
\hline
\end{tabular}

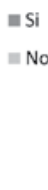

Figura 4. Presentación gráfica, resultados pregunta No. 4 
La respuesta a la pregunta número 4 se podría correlacionar con las respuestas a las preguntas 1 y 3 donde inicialmente la gran mayoría de estudiantes son los que responden a la encuesta y que en su mayoría son virtuales, es por eso que observamos cómo el $98 \%$ están de acuerdo con el BUV ya que permite más cobertura.

5. ¿Piensa usted que el bienestar universitario virtual promueve la visibilidad institucional de la universidad, a nivel nacional e internacional, convirtiéndose en un valor agregado para la misma?

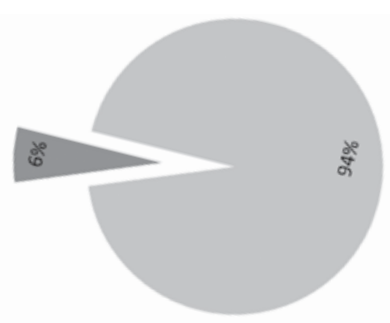

\begin{tabular}{|l|l|l|}
\hline $\mathrm{Si}$ & 231 & $94 \%$ \\
\hline No & 15 & $6 \%$ \\
\hline
\end{tabular}

Figura 5. Presentación gráfica, resultados pregunta No. 5

La respuesta a la pregunta 5 , muestra claramente como el $94 \%$ de la población cree que un BUV le puede ofrecer un mayor porcentaje de visibilidad a la universidad, lo que es algo muy interesante que quizá no ha sido evaluado por el personal administrativo, de ésta manera se podría recurrir a la opinión de los mismos estudiantes para que den su punto de vista sobre la visibilización de la universidad.

Estructura del Bienestar Universitario:

6. ¿De los siguientes actores cuales cree usted que deben intervenir en el proceso de Bienestar Universitario Virtual?

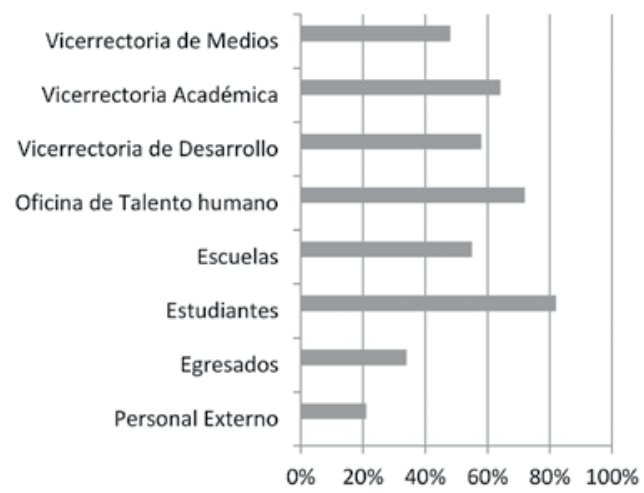




\section{Mauricio Perdomo Vargas y Víctor Fernando Cañón Rodríguez}

Bienestar universitario virtual, una estrategia para el fortalecimiento de la comunidad unadista con el uso de las TICs. artículo producto de la investigación

\begin{tabular}{|l|l|l|}
\hline Vicerrectoría de Medios & 119 & $48 \%$ \\
\hline Vicerrectoría Académica & 158 & $64 \%$ \\
\hline Vicerrectoría de Desarrollo & 142 & $58 \%$ \\
\hline Oficina de Talento Humano & 176 & $72 \%$ \\
\hline Escuelas & 136 & $55 \%$ \\
\hline Estudiantes & 22 & $82 \%$ \\
\hline Egresados & 83 & $34 \%$ \\
\hline Personal Externo & 52 & $21 \%$ \\
\hline
\end{tabular}

Figura 6. Presentación gráfica, resultados pregunta No. 6. Los usuarios podian seleccionar más de una casilla de verificación, por lo que los resultados superan el $100 \%$.

Considerando la estructura en esta pregunta apreciamos que en este proceso de intervención están con un $82 \%$ los estudiantes, seguido de la Oficina de Talento Humano (72\%) y con un 64\% la Vicerrectoría Académica; este dato muestra con una mayor incidencia a los estudiantes, aunque por logística y ofrecimiento de servicios esta la Oficina de Talento Humano en un segundo lugar, acompañada por las Vicerrectorías Académica y de Desarrollo (esta última con un 58\%). Por otra parte se crea un interrogante acerca del $21 \%$ que aparece dedicado al personal externo, en la medida que habría que revisar de qué forma puede intervenir este ente ante el bienestar de una universidad.

7. Según usted los objetivos del Bienestar Universitario Virtual deben estar orientados hacia:

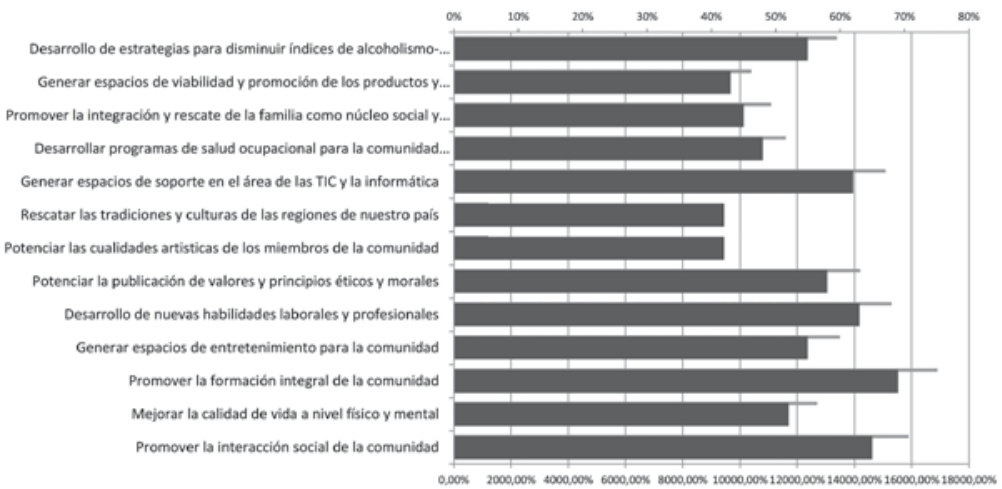

\begin{tabular}{|l|l|l|}
\hline Promover la interacción social de la comunidad & 159 & $65 \%$ \\
\hline Mejorar la calidad de vida a nivel físico y mental & 127 & $52 \%$ \\
\hline Promover la formación integral de la comunidad & 169 & $69 \%$ \\
\hline
\end{tabular}




\begin{tabular}{|c|c|c|}
\hline Generar espacios de entretenimiento para la comunidad & 135 & $55 \%$ \\
\hline Desarrollo de nuevas habilidades laborales y profesionales & 153 & $63 \%$ \\
\hline Potenciar la publicación de valores y principios éticos y morales & 142 & $58 \%$ \\
\hline Potenciar las cualidades artísticas de los miembros de la comunidad & 12 & $42 \%$ \\
\hline Rescatar las tradiciones y culturas de las regiones de nuestro país & 12 & $42 \%$ \\
\hline Generar espacios de soporte en el área de las TIC y la informática & 151 & $62 \%$ \\
\hline $\begin{array}{l}\text { Desarrollar programas de salud ocupacional para la comunidad } \\
\text { universitaria }\end{array}$ & 116 & $48 \%$ \\
\hline $\begin{array}{l}\text { Promover la integración y rescate de la familia como núcleo social y } \\
\text { gestor de valores }\end{array}$ & 111 & $45 \%$ \\
\hline $\begin{array}{l}\text { Generar espacios de viabilidad y promoción de los productos y servicios } \\
\text { de los actores de la comunidad académica }\end{array}$ & 104 & $43 \%$ \\
\hline $\begin{array}{l}\text { Desarrollo de estrategias para disminuir índices de alcoholismo } \\
\text { drogadicción y enfermedades infectocontagiosas }\end{array}$ & 134 & $55 \%$ \\
\hline
\end{tabular}

Figura 7. Presentación gráfica, resultados pregunta No. 7. Los usuarios podían seleccionar más de una casilla de verificación, por lo que los resultados pueden superan el 100\%.

En esta respuesta podemos observar que con los más altos porcentajes, el $69 \%$ y el 65\% (Anexos figura 2), la población da más importancia a una formación integral y a la interacción social, seguido de un $63 \%$ para el desarrollo de nuevas habilidades laborales y profesionales que se podría complementar con la primera elección de fortalecer la formación integral, luego con un $58 \%$ se da gran importancia a la publicación de valores éticos y morales; los demás ítems están sobre rangos del $40 \%$ encontrando con un menor porcentaje $(42 \%)$ la potenciación de cualidades artísticas, este último se pensaría inicialmente que hubiese sido el de más porcentaje pero se puede apreciar por parte de la comunidad un gran interés por la formación integral.

8. ¿Considera usted que dentro del sistema de BUV deben existir herramientas síncronas y asíncronas que permitan acceder a los servicios ofrecidos desde el sistema? 


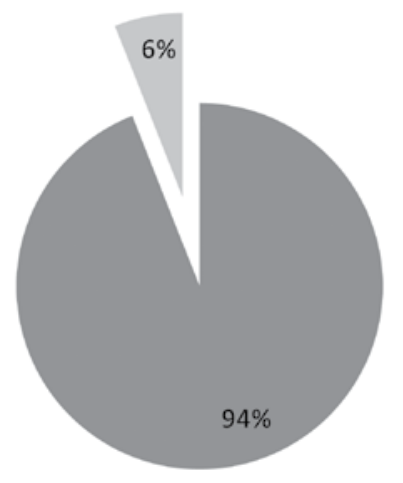

\begin{tabular}{|l|l|l|}
\hline Si & 231 & $94 \%$ \\
\hline No & 15 & $6 \%$ \\
\hline
\end{tabular}

Figura 8. Presentación gráfica, resultados pregunta No. 8

En la respuesta a la pregunta número 8 se puede observar que el 94\% ve de gran importancia la utilización de herramientas síncronas y asíncronas; se esperaría que fuera el $100 \%$ pero vemos que alcanza a percibirse un $6 \%$ que no considera importante la utilización de estas herramientas, sería interesante saber cuál es la razón para no verlas como viables.

9. ¿Considera que la comunidad en general (externo a la UNAD) debe tener acceso a algunos de los servicios que ofrece el bienestar universitario virtual?

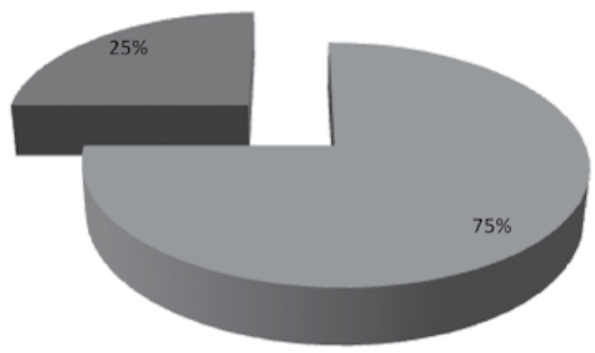

\begin{tabular}{|l|l|l|}
\hline $\mathrm{Si}$ & 184 & $75 \%$ \\
\hline No & 62 & $25 \%$ \\
\hline
\end{tabular}

Figura 9. Presentación gráfica, resultados pregunta No. 9

En la respuesta a la pregunta número 9 podemos observar que el $75 \%$ de la comunidad universitaria opina positivamente que personas externas a la universidad puedan acceder a algunos servicios del BUV, ahí es donde se podría pensar en espacios de compraventa o avisos publicitarios, en fin algo común para personal interno y externo; por su parte un $25 \%$ opinan que el BUV sea únicamente para personas que integren la universidad. 
10. Para usted el Sistema del Bienestar Universitario Virtual debe basarse en:

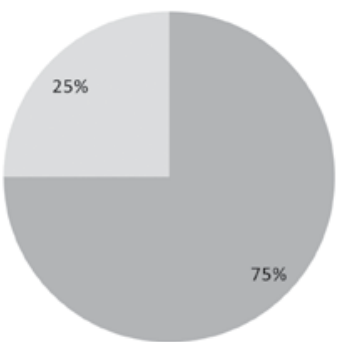

\begin{tabular}{|l|l|l|}
\hline $\begin{array}{l}\text { Programas } \\
\text { de alta } \\
\text { durabilidad }\end{array}$ & 184 & $75 \%$ \\
\hline $\begin{array}{l}\text { Servicios } \\
\text { de baja } \\
\text { duración }\end{array}$ & 62 & $25 \%$ \\
\hline
\end{tabular}

Figura 10. Presentación gráfica, resultados pregunta No. 10

Esta respuesta nos presenta un $75 \%$ de personas que opinan que los programas de Bienestar deben ser continuos y de alta durabilidad, en este sentido sería interesante evaluar en el actual bienestar como se están llevando a cabo los programas; por el contrario un $25 \%$ opinan que sería preferible que una oferta de múltiples programas y de baja duración.

11. De realizarse un sistema de Bienestar Universitario Virtual, usted como usuario le gustaría un sistema:

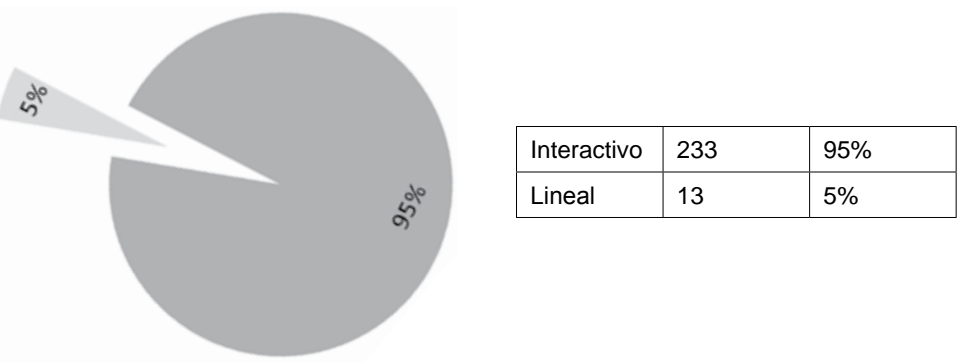

Figura 11. Presentación gráfica, resultados pregunta No. 11

Es importante ver que un 95\% piensa en un sistema BUV muy interactivo, esta pregunta también iría muy relacionada con la pregunta 3 , pues al utilizarse herramientas síncronas y asíncronas se tendría un buen sistema interactivo acompañado de diversas herramientas, un 5\% opinan que debería ser un sistema lineal; sería importante evaluar los motivos de este tipo de elecciones.

12. Del siguiente listado de formatos de los posibles contenidos del Bienestar Universitario Virtual, señale cuál o cuáles cree usted que son más propicios para presentar los contenidos en un sistema de bienestar universitario virtual. 


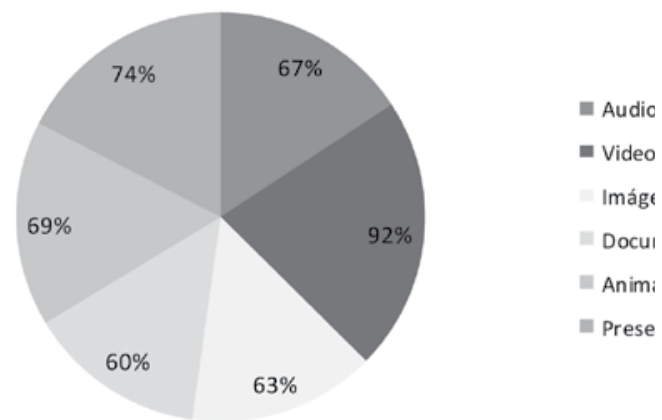

\begin{tabular}{|l|l|}
\hline Audio & 67 \\
\hline Video & 92 \\
\hline Imágenes & 63 \\
\hline Documentos & 60 \\
\hline Animaciones & 69 \\
\hline Presnetaciones & 74 \\
\hline
\end{tabular}

Figura 12. Presentación gráfica, resultados pregunta No. 12

En la respuesta a la pregunta número 12 podemos observar que un $92 \%$ de la población prefiere que su formación en los diferentes programas sea mediante videos, claro está que ninguno tiene menos de un $60 \%$, lo que indica que todas estas herramientas son importantes, pero curiosamente el $69 \%$ prefiere las animaciones, algo muy extraño ya que se podría afirmar que esta sería la más elegida; el que menos tiene con un $60 \%$ son los documentos, se puede percibir que aún hay una pasión por la lectura.

13. Según su criterio, ¿cree usted que un sistema de bienestar universitario virtual podría presentar debilidades o falencias en su estructura y desarrollo con la comunidad?

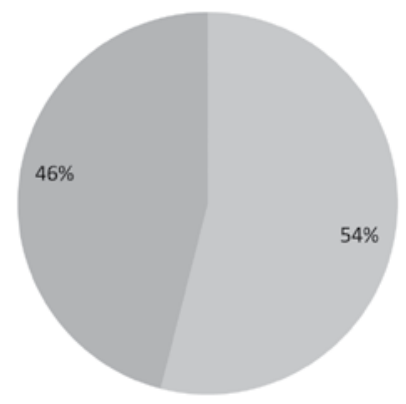

\begin{tabular}{|l|l|l|}
\hline Si & 134 & $54 \%$ \\
\hline No & 112 & $46 \%$ \\
\hline
\end{tabular}

Figura 13. Presentación gráfica, resultados pregunta No. 13

En cuanto a las debilidades encontramos porcentajes interesantes, donde hay un $54 \%$ que cree que se podrían presentar falencias en el desarrollo y estructura del sistema mientras que hay un $46 \%$ que opina que no se presentarán falencias, siendo un buen indicador para saber el buen funcionamiento de nuestra estructura en campus, lo que sería otro tema interesante para saber en qué se basa ese $54 \%$ para decir que sí se presentarían falencias. 
14. Del siguiente listado ¿cuál o cuáles serían las principales dificultades que se afrontarían si se ofrecieran servicios de bienestar universitario virtual? Marque 2 alternativas.

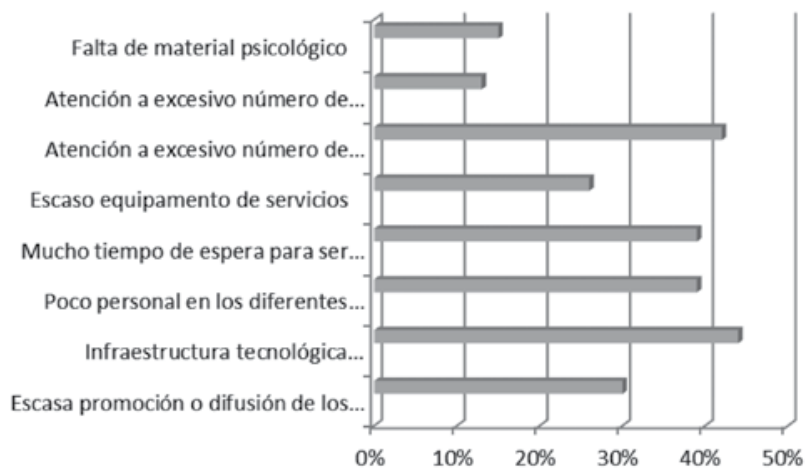

\begin{tabular}{|c|c|c|}
\hline $\begin{array}{l}\text { Escasa promoción o difusión de } \\
\text { los servicios del departamento }\end{array}$ & 73 & $30 \%$ \\
\hline $\begin{array}{l}\text { Infraestructura tecnológica } \\
\text { insuficiente e inadecuada }\end{array}$ & 108 & $44 \%$ \\
\hline $\begin{array}{l}\text { Poco personal en los diferentes } \\
\text { servicios }\end{array}$ & 96 & $39 \%$ \\
\hline $\begin{array}{l}\text { Mucho tiempo de espera para } \\
\text { ser atendido }\end{array}$ & 96 & $39 \%$ \\
\hline $\begin{array}{l}\text { Escaso equipamiento de } \\
\text { servicios }\end{array}$ & 63 & $26 \%$ \\
\hline $\begin{array}{l}\text { Atención a excesivo número de } \\
\text { estudiantes }\end{array}$ & 104 & $42 \%$ \\
\hline $\begin{array}{l}\text { Atención a excesivo número de } \\
\text { empleados }\end{array}$ & 31 & $13 \%$ \\
\hline Falta de material psicológico & 37 & $15 \%$ \\
\hline
\end{tabular}

Figura 14. Presentación gráfica, resultados pregunta No. 14

Otras variables marcadas por los encuestados relacionadas en el instrumento como Otras dificultades se presentan a continuación:

\begin{tabular}{|l|l|l|l|}
\hline Falta de planeación & 1 & $\begin{array}{l}\text { Poco personal en la } \\
\text { enseñanza de los } \\
\text { usuarios }\end{array}$ & 1 \\
\hline Ineficiencia & 1 & La plataforma & 1 \\
\hline $\begin{array}{l}\text { 3-Respuesta a solu- } \\
\text { ciones de bienestar de } \\
\text { cada CEAD }\end{array}$ & 1 & Organización & 1 \\
\hline
\end{tabular}




\begin{tabular}{|l|l|l|l|}
\hline Demasiados usuarios & 1 & $\begin{array}{l}\text { Poca inversión econó- } \\
\text { mica por parte de la } \\
\text { UNAD }\end{array}$ & 1 \\
\hline $\begin{array}{l}\text { Felicitaciones a las } \\
\text { directivas por esta } \\
\text { propuesta }\end{array}$ & 1 & $\begin{array}{l}\text { Demoras en la solución } \\
\text { de problemas }\end{array}$ & 1 \\
\hline $\begin{array}{l}\text { Que no contesten la } \\
\text { solicitud }\end{array}$ & 1 & $\begin{array}{l}\text { Desinterés por parte de } \\
\text { la comunidad }\end{array}$ & 1 \\
\hline $\begin{array}{l}\text { Falta de tiempo por } \\
\text { parte de los estudian- } \\
\text { tes }\end{array}$ & 1 & $\begin{array}{l}\text { Muchas actividades } \\
\text { habilitadas al mismo } \\
\text { tiempo }\end{array}$ & 1 \\
\hline $\begin{array}{l}\text { Escaso equipamiento } \\
\text { de servicios }\end{array}$ & 1 & $\begin{array}{l}\text { Falta de interés por } \\
\text { parte del estudiante }\end{array}$ & 1 \\
\hline Tiempo & 2 & Mal manejo & 1 \\
\hline $\begin{array}{l}\text { Solo pueden acceder } \\
\text { estudiantes de la } \\
\text { UNAD }\end{array}$ & 1 & Ninguna & 1 \\
\hline $\begin{array}{l}\text { Acceso a personal } \\
\text { externo de la UNAD }\end{array}$ & 1 & & \\
\hline
\end{tabular}

Es importante la lectura de las respuestas a esta última pregunta, donde vemos que de los posibles contratiempos propuestos para el sistema no hay ninguno que supere un $50 \%$, el máximo es de $44 \%$ donde se cree que una dificultad sería la infraestructura tecnológica, seguido de un $42 \%$ que indica que se presentarán dificultades en atender un excesivo número de estudiantes, seguido lo anterior de otros porcentajes que dan indicaciones pero no son tan relevantes. En otras circunstancias podemos apreciar cosas como falta de personal o de tiempo pero que no superan más de dos elecciones. Esto nos llevaría a pensar en la viabilidad del proyecto como eje fundamental en la formación académica. La encuesta se respondió entre el 23 de Octubre a las 3:36 pm hasta el 1 de Noviembre a las 18:16; siendo los días de mayor contacto el 24, 25 y 26 de octubre, con el pico más alto el 25 de octubre ( 75 encuestas contestadas).

\section{Discusión}

De acuerdo al análisis de resultados se puede iniciar esta discusión con una pregunta sencilla ¿Es vital para la UNAD la implementación de un sistema de bienestar universitario virtual?, respondiendo a esta pregunta creemos que sí, y se sustenta en los resultados hallados al aplicar el instrumento diseñado para este proyecto, donde se evidenció que el $98 \%$ de los miembros de la comunidad universitaria de la UNAD entrevistados, coinciden en la necesidad de implementar un sistema de bienestar en línea.

Diferentes variables inciden directamente en que este sistema de bienestar universitario virtual que en adelante llamaremos BUV, sea exitoso y cumpla con su principal objetivo: «Formar integralmente a la comunidad universitaria 
de la UNAD»; tanto en Colombia como en EEUU, ya que este sistema al estar desarrollado con las TICs, permite llegar en un entorno de alta virtualidad a diferentes contextos geográficos.

Para medir el éxito de BUV se hace necesario plantear un modelo estructural para el sistema y desde el mismo argumentar la necesidad de incorporar cada uno de los componentes que este debe poseer y que se relacionan a continuación como áreas estructurales del sistema, de esta forma se explicaran cada una de ellas a continuación, tomando como base inicial la gráfica siguiente que denota de forma clara cada una de estas.

\section{Conclusiones}

Los resultados obtenidos al indagar sobre un diagnóstico inicial sobre el bienestar universitario en la UNAD - Colombia, mediante el enfoque institucional, permiten evidenciar que las variables inciden de forma negativa ya que en la mayoría de los centros no disfrutan de un bienestar universitario de forma presencial. La metodología permitió analizar variables cuantitativas mediante indicadores que mostraron la reacción ante una propuesta de bienestar universitario.

Los datos y resultados tomados de diferentes centros de la UNAD permiten concluir que es urgente y necesaria una solución de bienestar universitario en línea para que todos puedan tener la oportunidad de visibilizar la universidad en su totalidad y poder tener acceso a este derecho.

No hay una relación de equidad entre todos los centros de la UNAD ya que los datos muestran que tan solo los nodos o centros grandes son los únicos que disfrutan de alguna forma de un bienestar universitario.

Los tipos de mediación (virtual-tradicional) en la UNAD son los que menos influyen en la no presencia de un bienestar en los centros, es más por causas institucionales ya que no se suministran los recursos para tal fin, por otra parte hay que tener en cuenta que en los centros pequeños el número de estudiantes no justifica un bienestar presencial, de esa manera se cuenta con un aval más para la implementación de un BUV.

\section{Referencias bibliográficas}

Consejo Nacional de Acreditación CNA. 2010. Lineamientos para acreditación institucional (Vol. serie No 2). Bienestar y ambiente institucional., www.cna.gov.co: (Consultado el 14 de Septiembre de 2010). 
Bienestar universitario virtual, una estrategia para el fortalecimiento de la comunidad unadista con el uso de las TICs. artículo producto de la investigación

Cotencha, L. F. 2008. Los sentidos del bienestar universitario en RudeColombia: Más allá de un servicio asistencial. Cali, http://www.ascun.org.co/.../Tesis\%20Doctoral\%20Luis\%20 F\%20Contecha\%20C.pdf, (Consultado el 10 septiembre de 2009).

Gallardo,Y.,yA.Moreno.1999. Modulo3Recoleccióndelainformación. EnICFES,Aprender a Investigar. Bogotá, http://www.calameo.com/books/0002428342a117e6b632c. (Consultado el 9 de Septiembre de 2010).

Garrido, E. 2003. Técnicas de recolección de datos. Venezuela: www.ucla.edu. ve/dmedicin/departamentos/.../recoleccion.pdf. (Consultado el 9 de Septiembre de 2010).).

Mayorga Rincón, O. Bienestar universitario. (U. C. Colombia, Ed.) Bogotá, http://www. ucc.edu.co/bogota/Paginas/Bienestaruniversitario.aspx: (última actualización 7 de Marzo de 2010) ((Consultado el 9 de Septiembre de 2010).

Peláez, M., y C. Ojeda. Perspectiva y prospectiva del bienestar universitario . $X X X$ Pleno Nacional de Bienestar Universitario ASCUN. http://www.ascun.org.co/ascun/ homeFiles/Experiencia\%20USTA.doc. (Consultado el 10 de Septiembre de 2009).

Restrepo. A, J. 2009. Nuevos Paradigmas de gestión universitaria y el bienestar universitario. XXX Pleno Nacional de Bienestar Universitario. http://www.ascun. org.co/ascun/homeFiles/Experiencia\%20USTA.doc. Paipa. (Consultado el 9 de Septiembre de 2010).

Romero, O. R. 2009. Ponencia Perspectivas y prospectivas de bienestar universitario. XXX Pleno Nacional de Bienestar Universitario ASCUN. http://www.ascun.org.co/ ascun/homeFiles/Experiencia\%20USTA.doc (Consultado el 10 de septiembre de 2009).

Universidad de Boyacá. Proyección universitaria. Bienestar Universitario. http://www. uniboyaca.edu.co/: (No registra última visita de actualización) (Consultado el 9 de Septiembre de 2010).

Universidad Digital. 2010. Libro Blanco. Ariel y Fundación Telefónica, http://www. universidaddigital2010.es/portal/page/udf/inici/publico (Consultado el 10 de septiembre de 2010).

Universidad Nacional de Colombia. 2006. «Relatoría de la Comisión de Bienestar Universitario, Facultad de Ingeniería». Bogotá. www.ing.unal.edu.co/representante/ documentos/mesa3_bienestar_total.pdf. (Consultado el 17 de Septiembre de 2010).Consultadohttp://www.uoc.edu/portal/castellano/la_universitat/index.html. (Consultado el (Consultado el 9 de Septiembre de 2010).

Vallejo Mejía. C. 2007. Políticas de bienestar desde las perspectivas del desarrollo humano. Pleno Nacional de Bienestar Universitario. Edición 28. Manizales: www. 
ascun.org.co/eventos/pleno2007/conferenciainaugural.pdf (Consultado el 9 de Septiembre de 2010).

Vara Horna, A. 2005. Capítulo 5 «Análisis de Datos». En La lógica de la investigación en las ciencias sociales. Perú. www.aristidesvara.com/libros/arch.../Logica_ Investigacion.pdf (Consultado el 9 de Septiembre de 2010). 